\title{
Tumour-Associated Retinal Pigment Epitheliopathy
}

\author{
B. E. DAMATO and W. S. FOULDS \\ Glasgow
}

\begin{abstract}
Summary
Choroidal tumours are associated with several degenerative changes in the overlying tissues, which can be called 'Tumour-Associated Retinal Pigment Epitheliopathy (TARPE)'. These changes include

(i) proliferation, detachment, atrophy, and metaplasia of the retinal pigment epithelium,

(ii) the accumulation of hard and soft drusen and basal laminar deposits in Bruch's membrane,

(iii) disorganisation of the choriocapillaris,

(iv) atrophy, cystic degeneration and detachment of the retina.

The changes at the chorio-retinal interface are clinically relevant because they can exacerbate visual loss. In addition, they can be misinterpreted on ophthalmoscopy and fluorescein angiography. An amelanotic choroidal tumour may appear to be pigmented on ophthalmoscopy because of lipofuscin and melanin accumulation overlying the tumour. The hyperfluorescence associated with pigmented choroidal melanomas is more likely to be related to degenerative changes in the retinal pigment epithelium than to dye leakage from abnormal tumour vessels.
\end{abstract}

Choroidal tumours cause a variety of abnormalities in the overlying choriocapillaris and retinal pigment epithelium. These changes,. which can be termed 'TumourAssociated Retinal Pigment Epitheliopathy (TARPE)', are clinically important because they can cause visual loss and because they may influence diagnosis and treatment. The aim of this paper is to highlight the main features of TARPE and to discuss their clinical significance.

\section{Clinico-pathological features of TARPE}

\section{Retinal Pigment Epithelium}

The retinal pigment epithelial cells overlying a choroidal melanoma tend to become dedifferentiated, losing apical processes and basal infoldings, and accumulating excessive amounts of intra-cytoplasmic lipofuscin.,
Mucoid inclusion bodies can also be present as in various reactionary processes associated with exudative retinal detachment. ${ }^{3}$ The retinal pigment epithelial cells undergo metaplasia, exhibiting the characteristics of fibroblasts or macrophages, and tend to form clumps and flattened papilliferous growths. ${ }^{1}$ As a result of these responses, the retinal pigment epithelium tends to be deficient in some areas and multilayered in others (Fig. 1).

Fluorescein angiography shows that retinal pigment epithelial atrophy tends to occur at the apex and the peripheral margin of the tumour, either in a crescentic fashion (Fig. 2i) or as a band running radially in the same direction as the underlying posterior ciliary arteries (Fig. 2). In addition, some eyes develop paving-stone degeneration peripheral to the tumour. ${ }^{4}$ 


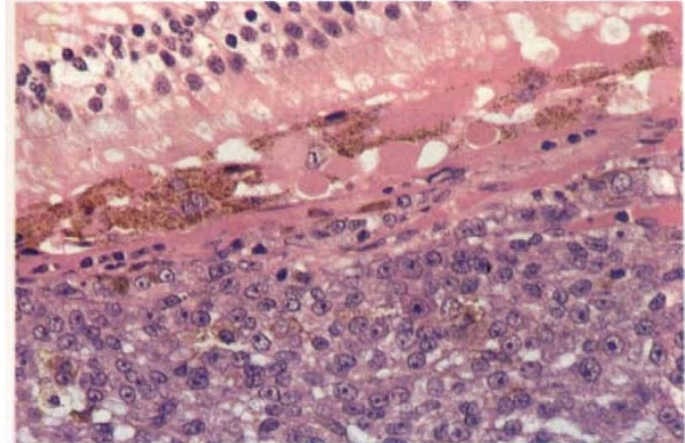

Fig. 1 (i).

Fig. 1. Changes in the retinal pigment epithelium and Bruch's membrane overlying choroidal melanomas. (i) loss of regularity of retinal pigment epithelium, choriocapillaris and outer retina, with hard drusen in Bruch's membrane ( $H \& E, \times 250)$. (ii) Soft drusen being invaded by macrophages, over a locally-resected choroidal melanoma. (Hence the absence of retina from the specimen). ( $H \& E, \times 150)$ (iii) Basal laminar deposits overlying a choroidal melanoma treated by local resection (Picro-Mallory stain, $\times 150)$. (iv) Retinal pigment epithelial detachments over a choroidal melanoma $(H \& E, \times 60)$.

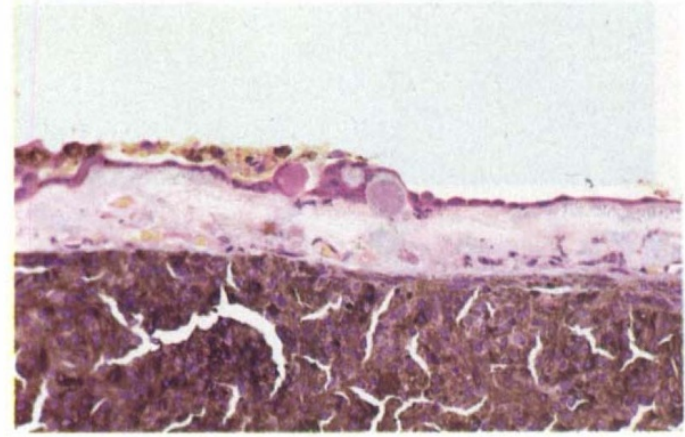

Fig. 1 (iii).

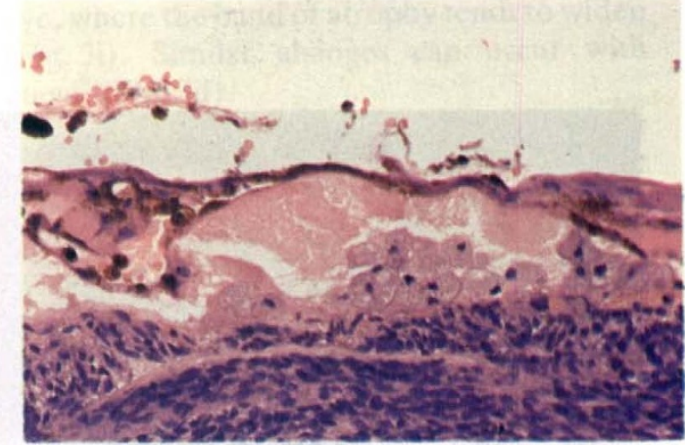

Fig. 1 (ii).

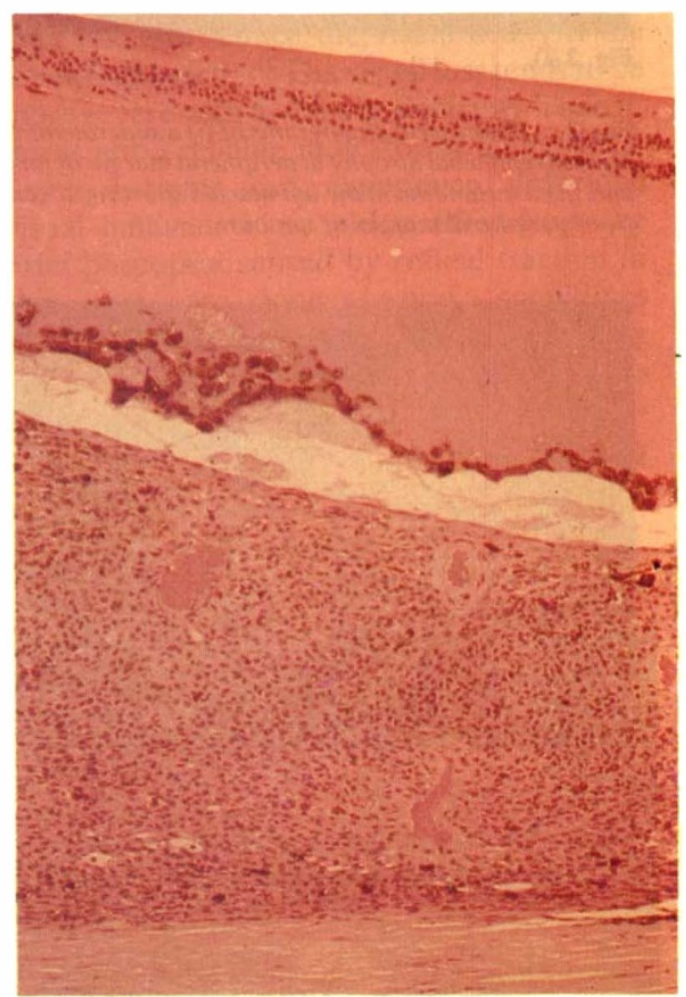

Fig. 1 (iv). 


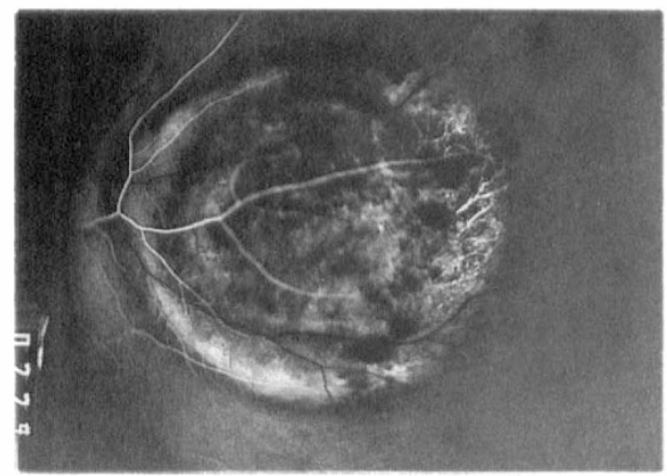

Fig. 2 (i).

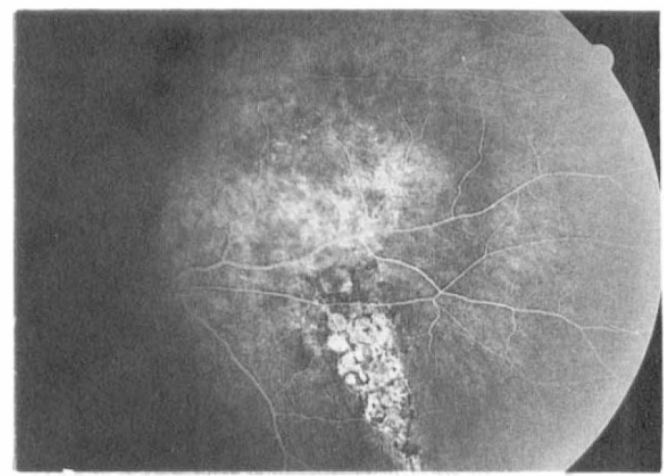

Fig. 2 (ii).

Fig. 2. Fluorescein angiograms of (i) a superotemporal choroidal melanoma (left eye), showing crescentic retinal pigment epithelial atrophy at peripheral margin of tumour with exposure of the underlying major choroidal vessels, and (ii) a melanoma at the left macula showing a 'comet's tail'-type of retinal pigment epithelial atrophy radiating from peripheral margin of tumour.

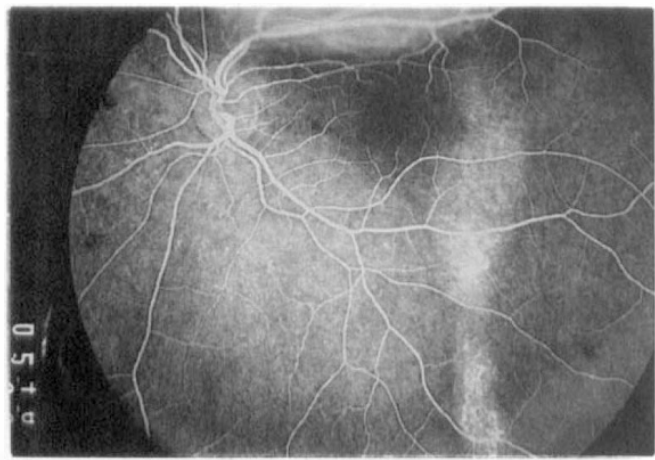

Fig. 3 (i).

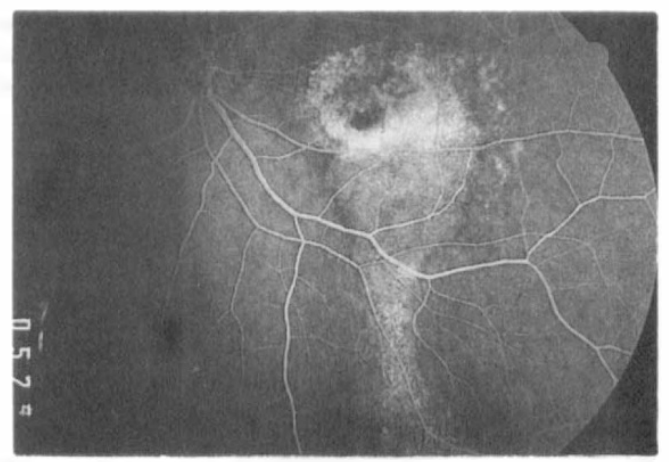

Fig. 3 (ii).

Fig. 3. Fluorescein angiographs of linear retinal pigment epithelial changes inferior to (i) a superior choroidal melanoma (left eye), and (ii) a choroidal naevus at the left macula. 


\section{Bruch's Membrane}

Bruch's membrane shows changes similar to those seen in age-related macular degeneration, ${ }^{5}$ with the formation of hard and soft drusen, and basal laminar deposits and pigment epithelial detachment (Fig. 1). Abnormal material in Bruch's membrane also attracts macrophages (Fig. 1) and stimulates the formation of choroidal new vessels, which may develop into clinically evident disciform lesions. ${ }^{6,7}$

\section{Blood Vessels}

The choriocapillaris is destroyed, especially at the tumour apex, (Fig. 1) and in the surviving capillaries the fenestrations are reduced in number. ${ }^{8}$ The major choroidal arteries seem to be unaffected in most cases, passing either through the tumour or over its surface to supply the peripheral choroid (Fig. 2).

\section{Retina}

Severe retinal pigment epithelial degener-

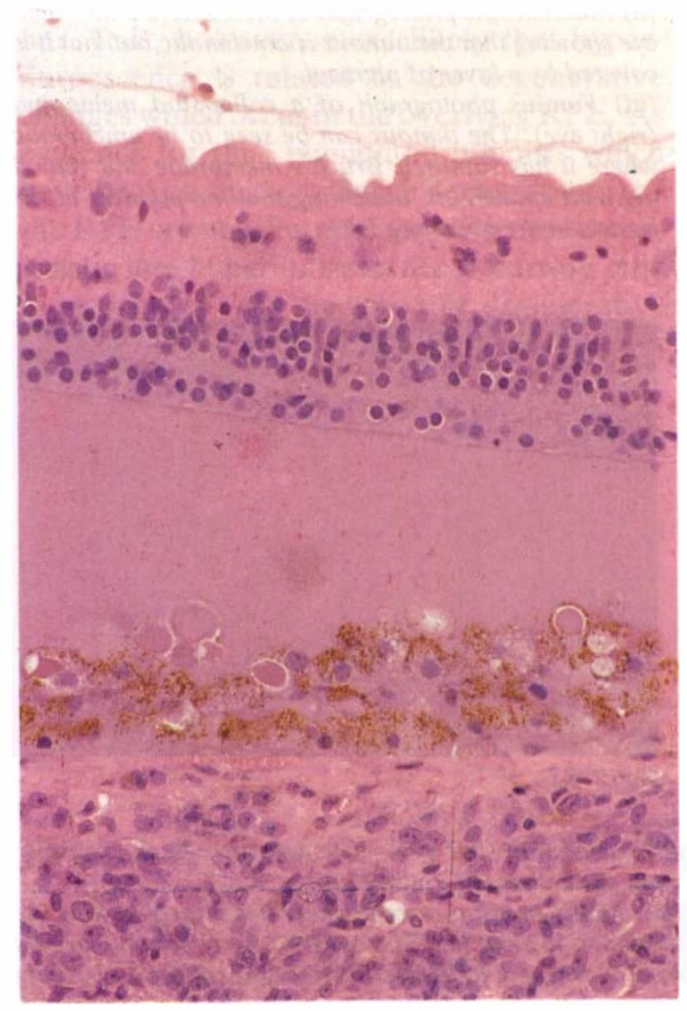

Fig. 4. Atrophy of the receptor cells in the detached retina overlying a choroidal melanoma $(H \& E, \times 250)$. ation is associated with serous retinal detachment, which usually extends beyond the limits of the tumour. A mild but long-standing detachment may cause a curvilinear band of RPE atrophy extending from the lower margin of the tumour to the inferior equator of the eye, where the band of atrophy tends to widen (Fig. 3i). Similar changes can occur with naevi ${ }^{9}$ (Fig. 3ii).

The retina overlying a choroidal tumour shows atrophy, commencing in the receptor cell layer, and gliosis (Fig. 4). Additionally, the retina may undergo cystic degeneration. Some patients develop cystoid macular oedema even if the tumour is situated equatorially (Fig. 5).

\section{Clinical Significance of TARPE}

\section{Visual Symptoms}

The changes in the retinal pigment epithelium and retina cause relative and absolute visual field loss and reduce the visual acuity if the fovea is affected. ${ }^{7,10}$ The visual loss tends to be more severe with melanomas than with naevi, but is not a reliable indicator of malignancy. Some patients can experience unformed visual hallucinations, which differ from the brief photopsia caused by retinal traction in that they are longer-lasting, sometimes moving across the visual field, and changing shape or dividing into smaller spots. It is interesting that these hallucinations can persist even after treatment of the tumour by radiotherapy or local resection.

\section{Ophthalmoscopic Appearances}

Choroidal tumours are often diagnosed as

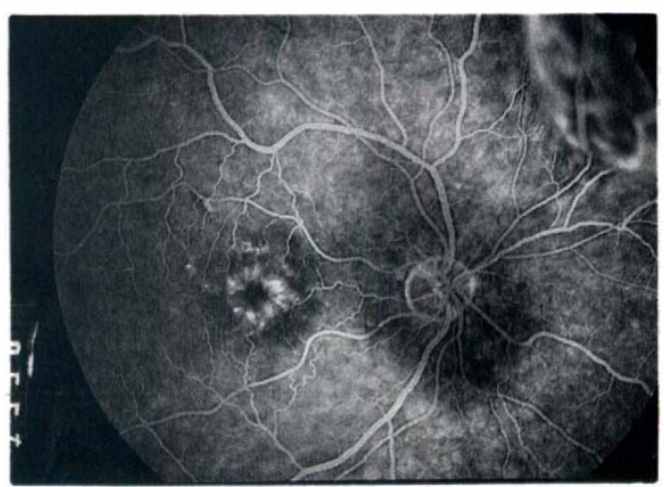

Fig. 5. Fluorescein angiograph of the right fundus showing cystoid macular oedema secondary to a superonasal choroidal melanoma. 


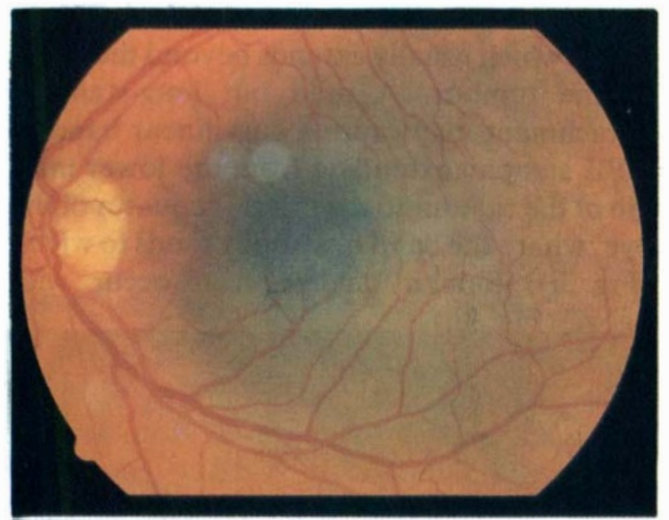

Fig. 6 (i).

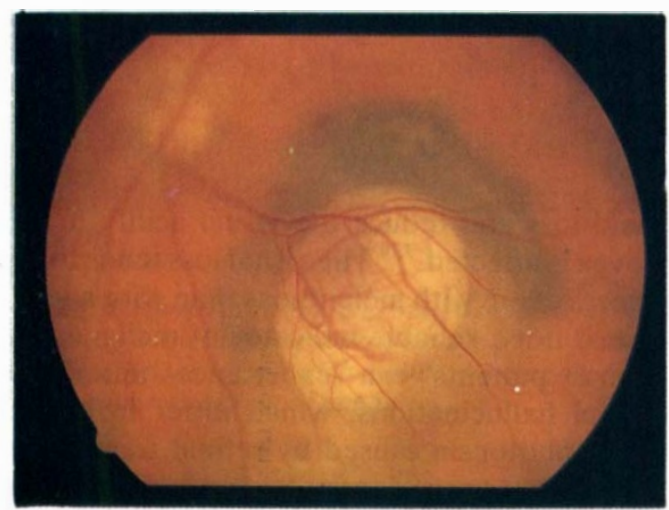

Fig. 6 (iii).

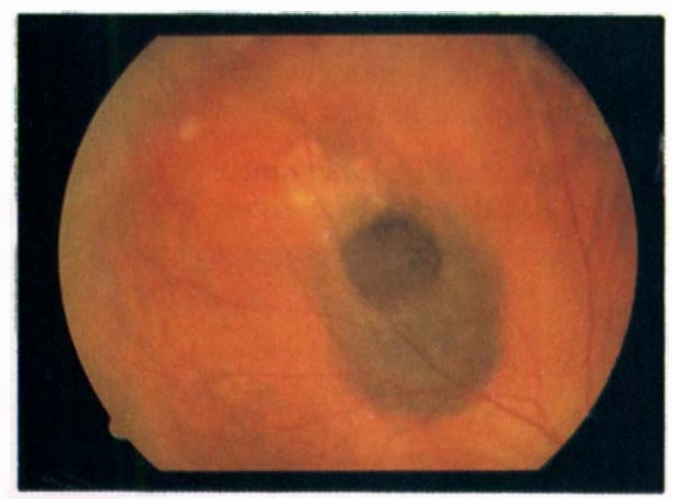

Fig. 7 (i)

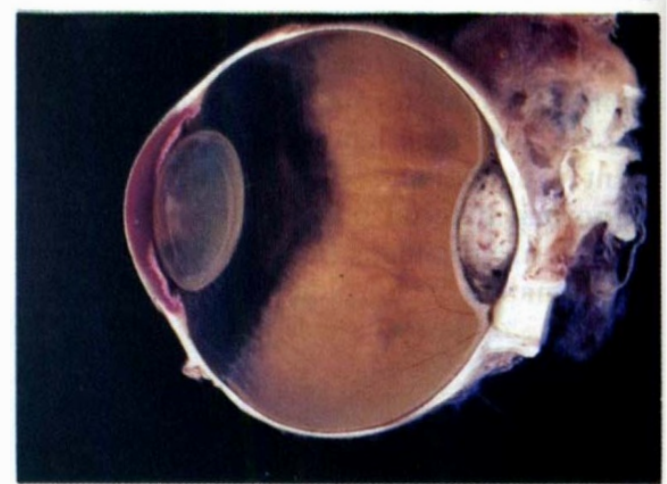

Fig. 6 (ii)

Fig. 6. (i) Fundus photograph of a choroidal melanoma in the right eye suggesting that the tumour is pigmented.

(ii) Macroscopic photograph of the bisected enucleated eye showing that the tumour is amelanotic but that it is covered by a layer of pigment.

(iii) Fundus photograph of a collar-stud melanoma (right eye). The tumour can be seen to be amelanotic where it has ruptured Bruch's membrane and retinal pigment epithelium, indicating that the pigment at the base is in the overlying RPE.

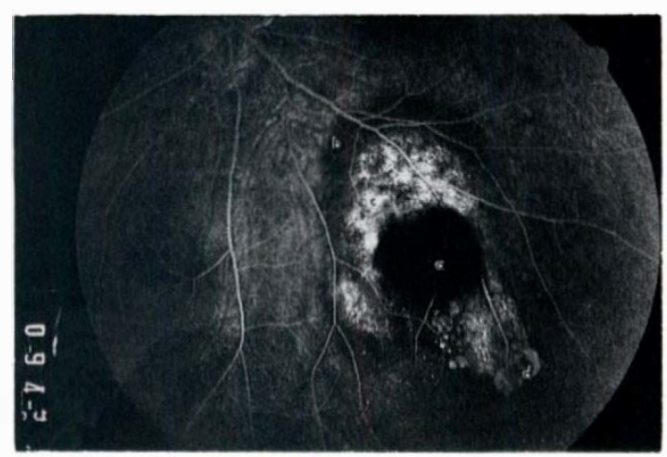

Fig. 7 (ii)

Fig. 7. (i) Fundus photograph of a pigmented choroidal melanoma (left eye). The tumour, which is deeply pigmented, has ruptured Bruch's membrane, retinal pigment epithelium and retina (a).

(ii) The fluorescein angiogram shows that the tumour itself is totally non-fluorescent where it is directly visible (a) and at its margins, where theoverlying retinal pigment epithelium is healthy $(b)$. The hyperfluorescence only occurs in areas where there is retinal pigment epithelial degeneration $(c)$ or where RPE atrophy has exposed the underlying sclera (d). 
melanomas because they appear to be pigmented on ophthalmoscopy. Nevertheless, most choroidal melanomas are amelanotic in caucasians and owe their pigmented appearance to the accumulation of lipofuscin and melanin over the surface of the tumour (Fig. 6).

Secondary degenerative changes overlying choroidal tumours tend to be a manifestation of chronicity and tumour bulk and are not specific to melanoma, occurring over large naevi, haemangiomas and other benign tumours. ${ }^{11}$

\section{Fluorescein Angiographic Appearances}

The characteristic angiographic appearances associated with malignant melanoma of the choroid are relative hypofluorescence in the early phase followed by the development of a patchy diffuse hyperfluorescence and discrete hyperfluorescent spots, which remain well defined throughout the angiogram. The late hyperfluorescence is generally assumed to be due to vascular incompetence within the tumour. It is, however, more likely that hyperfluroescence is related to the degenerative changes which occur in the overlying RPE. As evidence for this, pigmented tumours which have broken through Bruch's membrane and the RPE are non-fluorescent, with the characteristic late hyperfluorescence occurring only in association with recognisable degenerative changes in the RPE over the same tumour (Fig 7).

\section{Conclusion}

Many of the clinical features currently associated with malignant melanoma of the choroid are the result of secondary changes in the overlying tissues and are not specific either to melanoma or to malignancy. The recognition of TARPE is of value in the differential diagnosis of choroidal tumours.

We are grateful to Professor W. R. Lee for helpful advice and to Mrs. A. Currie for the artwork.

\section{References}

${ }^{1}$ Wallow IHL and Ts'O MOM: Proliferation of the retinal pigment epithelium over malignant choroidal tumors. Am J Ophthalmol 1972, 73: 914-26.

${ }^{2}$ Font RL, Zimmerman LE, Armaly MF: The nature of the orange pigment over a choroidal melanoma. Arch Ophthalmol 1974, 91: 359-62.

${ }^{3}$ Wolter JR. Cited in Duke-Elder S, Dobree JH, eds. System of Ophthalmology. Vol.X. Diseases of the retina. London: Henry Kimpton 1967: 34.

${ }^{4}$ Brown GC and Shields JA: Choroidal melanomas and paving stone degeneration. Ann Ophthalmol 1983, 15: 705-8.

${ }^{5}$ Bressler NM, Bressler SB, Fine SL: Age-related macular degeneration. Surv Ophthalmol 1988, 32: $375-413$.

${ }^{6}$ Lubin JR, Gragoudas ES, Albert DM: Choroidal neovascularisation associated with malignant melanoma: A case report. Acta Ophthalmol 1982, 60: 412-8.

${ }^{7}$ Gass JDM: Problems in the differential diagnosis of choroidal nevi and malignant melanomas. The XXXIII Edward Jackson Memorial Lecture. Am J Ophthalmol 1977, 83: 299-323.

${ }^{8}$ Federman JL: The fenestrations of the choriocapillaris in the presence of choroidal melanoma. $\mathrm{Tr}$ Am Ophthalmol Soc 1982, 80: 498-516.

${ }^{9}$ Haut J, Sobel-Martin A, Dureuil J, Larricart P, Sarnikowski C: Atrophies 'like flows' of the retinal pigment epithelium: A neuroepithelium-draining method of the posterior pole. Ophthalmologica 1984, 189: 121-7.

${ }^{10}$ Gonder JR, Augsburger JJ, McCarthy EF, Shields JA: Visual loss associated with choroidal nevi. Ophthalmology 1982, 89: 961-5.

${ }^{11}$ Shields JA, Rodrigues MM, Sarin LK, Tasman WS, Annesley WH: Lipofuscin pigment over benign and malignant choroidal tumors. Trans Am Acad Ophthalmol Otolaryngol 1976, 81: 871-81. 